\title{
Traveling fronts in systems of particles with random velocities
}

\author{
Vicenç Méndez, Daniel Campos, and Ignacio Gómez-Portillo \\ Grup de Física Estadística, Departament de Física, Facultat de Ciències, Universitat Autònoma de Barcelona, \\ Bellaterra, Barcelona 08193, Spain
}

(Received 28 July 2010; revised manuscript received 1 October 2010; published 21 October 2010)

\begin{abstract}
We explore a reaction-dispersal mechanism for the generation of wave fronts which consists of a set of particles traveling with random velocities (chosen from arbitrary distributions) which experience an autocatalytic reaction. The differences found between this mechanism and approaches based on the continuous-time random walks, where the particles are assumed to perform discrete jumps from one position to another, are analyzed and discussed. A complete analytical treatment of our velocity model is achieved, which allows us to predict the constant speed of traveling fronts or their time dependence in case they are accelerated. Also, a general criterion to distinguish the situations of fronts with constant speed from those accelerated is provided. From it, we conclude that accelerated fronts should be expected in different situations easily achievable in nature.
\end{abstract}

DOI: 10.1103/PhysRevE.82.041119

PACS number(s): 05.40.Fb, 02.50.Ey, 05.45.-a

\section{INTRODUCTION}

Reaction-dispersal models represent an archetypical framework to understand and implement spread phenomena and traveling solutions, in general, e.g., fronts, pulses, or waves. This has found application in a wide variety of fields like in chemical waves and patterns [1,2], biological [3,4] and human [5] invasions, epidemics propagation [6], tumor growth [7], and so on.

Classical reaction-dispersal equations are built on the hypothesis that individual particles move according to Fickian diffusion, so one typically refers then to "reaction-diffusion" systems. Such diffusive transport can be implemented by considering that particles either "jump" from one position to another or move with constant velocity, provided in both cases that the characteristic time scale of the transport process is very small compared to the observation time or the other time scales in the system. Thus, the potential effects of long-range dispersal (plus other microscopic details of the transport pattern) are lost within that approach. This restriction can be relaxed by introducing the well-known idea of continuous-time random walks (CTRWs). Within the CTRW framework, particles can jump from one position to another with an arbitrary distribution of jump lengths, these jumps being separated by waiting times also randomly distributed. This generalized mechanism allows one to implement, among others, transport patterns that exhibit anomalous diffusion. These are characterized by the power-law scaling $t^{\alpha}$ for the mean-square displacement, with $\alpha \neq 1$ ( $\alpha=1$ for Fickian diffusion). Actually, several authors have used this idea in the past years to explore the properties of traveling wave solutions and wave fronts for reaction-dispersal systems under the condition that the dispersal process on its own corresponds to subdiffusion $(\alpha<1)$ [8-11].

However, the idea that particles travel through discrete jumps from one position to another is only realistic for some specific situations, like in compartmentalized media (where particles move effectively just from one compartment to another) or patchy environments [12]. On the contrary, many systems in nature (for instance, cell cultures or chemical re- actions mediated by transport in a sparse medium) fit much better a velocity-based approach. For those cases, velocity models, where individual particles move continuously with a given velocity instead of making jumps, become extremely useful. The simplest versions of such velocity models assume that the speed of a particle takes a fixed value $v_{0}$, while only the direction of movement can change in time. For the one-dimensional (1D) case where the particle can choose only between values $v_{0}$ or $-v_{0}$ with probability $1 / 2$, and the change from positive velocity to negative (and vice versa) is governed by a Poisson process with characteristic rate $\beta$, one obtains the so-called hyperbolic reaction-diffusion equation $[1,13-15]$. The generalization to the case where the times between consecutive changes in the direction follow an arbitrary probability distribution (that is, they are not simply determined by a constant rate $\beta$ ) was provided by Zumofen and Klafter [16]. The composite case where the particles can travel with a fixed speed while at the same time they experience Fickian diffusion (which holds, for example, in a system of self-propelled particles influenced by thermal noise) has been studied in [17-19]. Finally, the situation where sojourns with a fixed velocity $v_{0}$ are separated by waiting periods (which has special interest in the field of cellular migration) has been explored in recent works [20].

An important advance within this context has been provided by Zaburdaev et al. [21]. They have shown how to extend the idea of the standard CTRW to the case where the individual particles can travel with an arbitrary distribution of velocities instead of making jumps. Their original work and a recently published continuation [22], however, just focused on the properties of the transport process, while the effects resulting from a reaction (that is, a birth-death) mechanism have not been considered yet. The present work tries to fill that gap by exploring the characteristic traveling fronts arising from the random velocity model presented in [21] when a reaction term is considered. As we shall see, the properties of these fronts show some interesting differences if compared with those models based on the idea of "jumps." For example, we find that accelerated fronts emerge for some velocity distributions which are not heavy tailed. This is in contrast with the CTRW model and all reaction-dispersal 
models based on jumps, where a very slowly decaying distribution function of jump lengths (typically slower than exponential) is necessary to obtain such accelerated solutions $[23,24]$. Also, we show in this work that the asymptotic equivalence found by Zaburdaev et al. between their model and the CTRW breaks down when the reaction process is implemented; this will become evident by proving that the characteristic speed of the fronts found in the two cases is different.

This paper is organized as follows. The CTRW framework and its random-velocity counterpart are presented in Sec. II. In Sec. III we derive a criterion for distinguishing the cases where reaction-dispersal fronts with constant speed or accelerated are obtained. Different analytical results for the characteristic speed and the asymptotic behavior of these fronts are provided for both cases (Secs. III and VI). These results will also be compared to numerical simulations for different cases of special interest. Finally, the conclusions of our work will be left for Sec. V.

\section{CTRW AND MODEL WITH RANDOM VELOCITIES}

Let us consider for simplicity an infinite 1D continuous medium in which a set of noninteracting particles can jump from one position to another according to the jump length probability distribution $\Phi(x)$. Before they jump, particles have to wait at their current position for a random time distributed according to $\varphi(t)$. Then, if we define $J_{j}(x, t)$ as the density of particles arriving to the position $x$ at time $t$, we have

$$
\begin{aligned}
J_{j}(x, t)= & \int_{0}^{t} d t^{\prime} \int_{-\infty}^{\infty} d x^{\prime} J_{j}\left(x-x^{\prime}, t-t^{\prime}\right) \varphi\left(t^{\prime}\right) \Phi\left(x^{\prime}\right) \\
& +J(x, 0) \delta(t),
\end{aligned}
$$

where the subindex $j$ stands for jumps and the second term in the right-hand side represents the contribution from the initial condition. For the sake of clarity, let us mention that sometimes these initial conditions are introduced, alternatively, through a term of the type $\int_{-\infty}^{\infty} d x^{\prime} P(x$ $\left.-x^{\prime}, 0\right) \varphi(t) \Phi\left(x^{\prime}\right)$, so defining an initial density of particles $P(x, 0)$ (see, for example, Section 3.4 in Ref. [1]). The difference between this term and the one used in Eq. (1) reflects just the technical question of whether the particles are "put at position $x$ and then the time is reset" at $t=0$ [then it is more natural to define an initial density $P(x, 0)]$ or "first the clock is reset at $t=0$ and then the particles are put there" [leading to $J(x, 0)]$. In any case, this is just a minor point which does not change at all the form of the differential master equation [see Eq. (3) below] or the global dynamics of the system, and obviously it does not affect the results for traveling fronts presented here.

According to expression (1), the density of particles standing at the position $x$ at the time $t$ will be computed through

$$
P_{j}(x, t)=\int_{0}^{t} d t^{\prime} J_{j}\left(x, t-t^{\prime}\right) \phi\left(t^{\prime}\right),
$$

where $\phi(t)=\int_{t}^{\infty} d t^{\prime} \varphi\left(t^{\prime}\right)$ is the "survival" probability distribution of $\varphi(t)$; i.e., it gives us the probability that the particle has not jumped yet after a waiting period of duration $t$ standing at its current position.

Equations (1) and (2) represent the cornerstone of the CTRW model. By inserting the solution of Eq. (1) into Eq. (2) and differentiating with respect to time one finds

$$
\begin{aligned}
\frac{\partial P_{j}(x, t)}{\partial t}= & \int_{0}^{t} d t^{\prime} \int_{-\infty}^{\infty} d x^{\prime} P_{j}\left(x-x^{\prime}, t-t^{\prime}\right) M\left(t^{\prime}\right) \Phi\left(x^{\prime}\right) \\
& -\int_{0}^{t} d t^{\prime} P_{j}\left(x, t-t^{\prime}\right) M\left(t^{\prime}\right),
\end{aligned}
$$

which is commonly known as the CTRW master equation. The memory kernel $M(t)$ is defined through its Laplace transform in time (denoted by $\hat{\text { ) }}$,

$$
\hat{M}(s)=\frac{s \hat{\varphi}(s)}{1-\hat{\varphi}(s)},
$$

where $s$ is the corresponding argument.

Now we will introduce a reaction process into this framework. Since we are interested here in the analysis of wave fronts propagating into unstable states, we will consider an autocatalytic process of the type $\mathrm{A}+\varnothing \rightarrow 2 \mathrm{~A}$, which is one of the simplest mechanisms able to introduce such selfsimilar solutions. Defining $r$ as the characteristic parameter of the reaction process, we have that the density $R(x, t)$ of particles appearing from the reaction process can be written as a logistic growth term

$$
R(x, t)=r P(x, t)[1-P(x, t)]
$$

where $r P(x, t)$ and $-r P(x, t)^{2}$ stand for the birth and mortality terms, respectively (where the latter term has been introduced to avoid unbounded growth of the particle density). In Eq. (5) we have not specified the subindex for $P$ because this relationship holds both for jump and velocity models. We can implement reaction term (5) into the CTRW if we assume that the new particles created with the rate $r P(x, t)$ have zero age (see Section 3.4.2 in Ref. [1] for further details). Then, Eqs. (1) and (2) turn into

$$
\begin{gathered}
J_{j}(x, t)=\int_{0}^{t} d t^{\prime} \int_{-\infty}^{\infty} d x^{\prime} J_{j}\left(x-x^{\prime}, t-t^{\prime}\right) \varphi\left(t^{\prime}\right) \Phi\left(x^{\prime}\right) \Omega_{j}(x \\
\left.-x^{\prime}, t, t^{\prime}\right)+J_{j}(x, 0) \delta(t)+r P(x, t) \\
P_{j}(x, t)=\int_{0}^{t} d t^{\prime} J_{j}\left(x, t-t^{\prime}\right) \phi\left(t^{\prime}\right) \Omega_{j}\left(x, t, t^{\prime}\right)
\end{gathered}
$$

where 

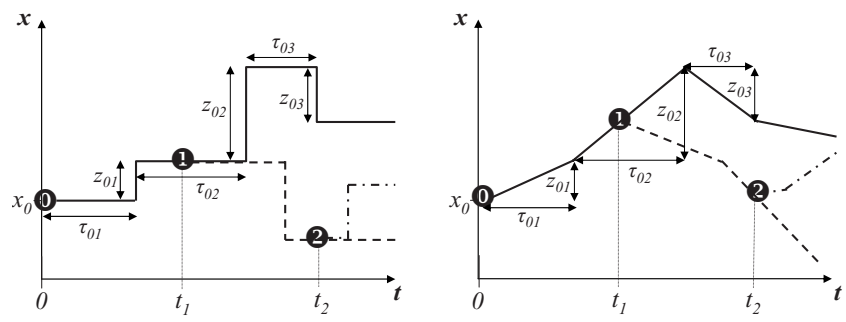

FIG. 1. Schematic representation of the trajectories of particles in the reaction-dispersal mechanism for the CTRW (left) and its velocity counterpart presented here (right). The balls with the number inside represent individual particles at the moment when they appear in the system, and so their dynamics starts. The $x$ - $t$ trajectories of the particles are represented by solid lines (particle 0), dashed lines (particle 1), and dashed-dotted lines (particle 2). The values of $\tau_{01}, \tau_{02}, \ldots$ and $z_{01}, z_{02}, \ldots$ correspond to the waiting (flight) times and the jump (flight) lengths of the particle 0 , respectively.

$$
\Omega_{j}\left(x, t, t^{\prime}\right) \equiv \exp \left(-r \int_{t-t^{\prime}}^{t}\left[P_{j}(x, u)\right]^{2} d u\right)
$$

is a survival probability term which takes into account the effects of the nonlinear (mortality) term in Eq. (5).

Note that alternative forms to Eqs. (6) and (7) have also been proposed in the literature to implement nonlinear kinetic terms (see [9] and Sections 3.4.1 and 3.4.3 in [1]). Specifically, careful choice of this equation is necessary (since it can lead to dramatically different results) in the case of heavy-tailed waiting time distributions since the system shows effects such as aging or ergodicity breaking which strongly affect its general dynamics $[8,9,11]$.

The trajectory scheme of the particles experiencing a CTRW plus reaction as that described above is depicted in Fig. 1 (left). The figure depicts only the linear part of the reaction term (birth process), as mortality processes underlying the nonlinear term can be argued to be irrelevant for the speed of the traveling front (see Secs. IV and V). The solid lines there represent the trajectory of the particle " 0 ," initially standing at position $x_{0}$, whose waiting times between jumps are denoted by $\tau_{01}, \tau_{02}, \ldots$, while $z_{01}, z_{02}, \ldots$ represent its corresponding jump lengths. Also, the particle " 0 " is seen to generate, at a given time $t_{1}$, a new particle " 1 " which then starts its independent dynamics and so on. The trajectory followed by each particle is drawn in a different line style (see the figure caption). Figure 1 (right), on the contrary, represents the equivalent situation for the case in which the particles do not wait and jump from one position to another but move continuously with a certain random velocity. The velocity of the particle remains constant during a certain "flight time" and then it is given a new value randomly. Note that, in order to facilitate the comparison, the jump lengths and waiting times in the CTRW scheme (left) are chosen equal to the distances covered by the particles and the corresponding "flight times" in the velocity scheme (right).

The scheme in the right of Fig. 1 corresponds to the velocity counterpart of the CTRW introduced by Zaburdaev et al. [21], plus the reaction (birth) process implemented as discussed above. Following the prescriptions in the original reference, we can define $h(v)$ as the probability distribution that the particle performs a single "flight" with velocity $v$. Then the velocity model (with reaction) will be governed by reaction (5) together with

$$
\begin{gathered}
J_{v}(x, t)=\int_{0}^{t} d t^{\prime} \int_{-\infty}^{\infty} d v J_{v}\left(x-v t^{\prime}, t-t^{\prime}\right) \varphi\left(t^{\prime}\right) h(v) \Omega_{v}(x \\
\left.-v t^{\prime}, t, t^{\prime}\right)+\delta(x) \delta(t)+r P_{v}(x, t), \\
P_{v}(x, t)=\int_{0}^{t} d t^{\prime} \int_{-\infty}^{\infty} d v J_{v}\left(x-v t^{\prime}, t-t^{\prime}\right) \phi\left(t^{\prime}\right) h(v) \Omega_{v}\left(x, t, t^{\prime}\right),
\end{gathered}
$$

where the subindex $v$ stands for "velocity." Note that Eqs. (8) and (9) are formally very similar to Eqs. (6) and (7); however, it must be stressed that the specific meaning of the densities is different in both cases. Here, $J_{v}$ represents the density of particles that finish a single flight (that is, they change the value of their speed) at time $t$ at position $x$. Likewise, $P_{v}$ is the density of particles passing through position $x$ at time $t$.

If we compare the two schemes in Fig. 1, it can be seen that the position of the particle 0 coincides at times $\tau_{01}, \tau_{01}$ $+\tau_{02}, \ldots$ albeit the trajectory of the particle is different in each case. This implies that the asymptotic transport properties (without considering reaction) of the CTRW model and its velocity counterpart will be equivalent. Actually, Zaburdaev et al. gave the relation [21]

$$
\Phi(x)=\int_{0}^{\infty} d t \int_{-\infty}^{\infty} d v \delta(x-v t) \varphi(t) h(v)
$$

between the jump length distribution in the CTRW and the velocity distribution in their model, which ensures the asymptotic equivalence between both approaches.

We will show that this equivalence between jumps and velocities, which is valid when only transport is being considered, breaks down when the reaction is introduced. Actually, this is not an effect of the reaction process on its own. Equation (10) guarantees that the asymptotic statistical properties of both models coincide, but it is also true that the density profiles are different in jump and velocity models, as has been emphasized previously [22]. What the reaction process does is to strengthen these differences, which makes the macroscopic dynamics of the system different too, as we shall see.

From Fig. 1 it is also easy to understand intuitively how these differences between jump and velocity models emerge. While the behavior of the particle " 0 " will be the same asymptotically in both cases, the particle " 1 " is created at position $x_{0}+z_{01}$ in the CTRW scheme, but it appears forward in the velocity scheme (and the same will happen, in general. for particles " 2 ," " 3 ," etc.). As a result, one must expect that the characteristic speed of traveling solutions obtained from this reaction-dispersal framework will be always faster in the 
velocity scheme than in the CTRW scheme, provided equivalence condition (10) is fulfilled. This is exactly what one finds (see Sec. IV).

\section{CRITERION FOR THE SPEED OF TRAVELING FRONTS}

The jump length distribution (known as dispersal kernel in ecological literature) given in Eq. (10) allows us to know whether the front will propagate with constant speed or will accelerate. Given $\varphi(t)$ and $h(v)$, if the resulting $\Phi(x)$ has finite moments and its moment-generating function is also finite then the front will propagate with constant speed. This criterion has been developed and discussed in detail for the case of jumps in [25]. Note that according to this, if the moment-generating function diverges (even if the moments are finite) then the front will accelerate; some examples of this situation are provided below. Let us define the moments of the PDFs, $\Phi(x), \varphi(t)$, and $h(v)$, as

$$
\begin{gathered}
\left\langle x^{n}\right\rangle=\int_{-\infty}^{\infty} x^{n} \Phi(x) d x, \\
\left\langle t^{n}\right\rangle=\int_{0}^{\infty} t^{n} \varphi(t) d t, \\
\left\langle v^{n}\right\rangle=\int_{-\infty}^{\infty} v^{n} h(v) d v,
\end{gathered}
$$

respectively. From Eq. (10), it is not difficult to show that the moments of the distribution read

$$
\begin{aligned}
\left\langle x^{n}\right\rangle & =\int_{-\infty}^{\infty} x^{n} \Phi(x) d x \\
& =\int_{-\infty}^{\infty} x^{n} d x \int_{0}^{\infty} d t \int_{-\infty}^{\infty} d v \delta(x-v t) \varphi(t) h(v) \\
& =\int_{0}^{\infty} d t^{\varphi} \frac{\varphi(t)}{t} \int_{-\infty}^{\infty} x^{n} h\left(\frac{x}{t}\right) d x=\int_{0}^{\infty} d t \varphi(t) t^{n} \int_{-\infty}^{\infty} z^{n} h(z) d z \\
& =\left\langle t^{n}\right\rangle\left\langle v^{n}\right\rangle .
\end{aligned}
$$

The moment-generating function $\Phi(p)=\int_{-\infty}^{\infty} \Phi(x) e^{p x} d x$ can be expressed as the sum

$$
\Phi(p)=\sum_{n=0}^{\infty} \frac{p^{n}}{n !}\left\langle t^{n}\right\rangle\left\langle v^{n}\right\rangle .
$$

As a result, if this series does not converge then the momentgenerating function does not exist; so, if $\left\langle t^{n}\right\rangle\left\langle v^{n}\right\rangle$ grows with $n$ faster than $n ! a^{n}$ then the front will accelerate, with $a$ as a certain constant. This requirement is a general condition to have accelerated fronts. In the following, we will show how to compute the front speed when it is constant or accelerated.

\section{FRONTS PROPAGATING WITH CONSTANT SPEED}

To observe traveling fronts in reaction-dispersal systems it is necessary to choose appropriate initial conditions (conditions with compact support are often required [26]); for the sake of simplicity we will consider here always $J_{v}(x, 0)$ $=\delta(x)$. When the criterion provided in Sec. III predicts front traveling with constant speed, this speed can be computed with the Hamilton-Jacobi formalism [1]. This method consists, first of all, in inserting the hyperbolic scaling $x \rightarrow x / \varepsilon$ and $t \rightarrow t / \varepsilon$ with $\varepsilon \ll 1$ into Eqs. (8) and (9) to get

$$
\begin{gathered}
J_{v}^{\varepsilon}(x, t)=\int_{0}^{t / \varepsilon} d t^{\prime} \int_{-\infty}^{\infty} d v J_{v}^{\varepsilon}\left(x-\varepsilon v t^{\prime}, t-\varepsilon t^{\prime}\right) \varphi\left(t^{\prime}\right) h(v) \Omega_{v}\left(\frac{x}{\varepsilon}-v t^{\prime}, \frac{t}{\varepsilon}, t^{\prime}\right)+\delta(x / \varepsilon) \delta(t / \varepsilon)+r P_{v}^{\varepsilon}(x, t), \\
P_{v}^{\varepsilon}(x, t)=\int_{0}^{t / \varepsilon} d t^{\prime} \int_{-\infty}^{\infty} d v J_{v}^{\varepsilon}\left(x-\varepsilon v t^{\prime}, t-\varepsilon t^{\prime}\right) \phi\left(t^{\prime}\right) h(v) \Omega_{v}\left(\frac{x}{\varepsilon}, \frac{t}{\varepsilon}, t^{\prime}\right),
\end{gathered}
$$

where we have defined the new fields $J_{v}^{\varepsilon}(x, t)=J_{v}(x / \varepsilon, t / \varepsilon), P_{v}^{\varepsilon}(x, t)=P_{v}(x / \varepsilon, t / \varepsilon)$, and

$$
\Omega_{v}\left(\frac{x}{\varepsilon}, \frac{t}{\varepsilon}, t^{\prime}\right) \equiv \exp \left(-\frac{r}{\varepsilon} \int_{t-\varepsilon t^{\prime}}^{t}\left[P_{v}^{\varepsilon}(x, u)\right]^{2} d u\right) .
$$

Since $J_{v}^{\varepsilon}(x, t)$ and $P_{v}^{\varepsilon}(x, t)$ are positive we can make use of the transformations $J_{v}^{\varepsilon}(x, t)=A_{1} e^{-G^{\varepsilon}(x, t) / \varepsilon}$ and $P_{v}^{\varepsilon}(x, t)=A_{2} e^{-G^{\varepsilon}(x, t) / \varepsilon}$, which when inserted into Eq. (12) give

$$
\begin{gathered}
A_{1}=A_{1} \int_{0}^{t / \varepsilon} d t^{\prime} \int_{-\infty}^{\infty} d v \exp \left[v t^{\prime} \partial_{x} G^{\varepsilon}+t^{\prime} \partial_{t} G^{\varepsilon}+O(\varepsilon)\right] \varphi\left(t^{\prime}\right) h(v) \Omega_{v}\left(\frac{x}{\varepsilon}-v t^{\prime}, \frac{t}{\varepsilon}, t^{\prime}\right)+\delta(x / \varepsilon) \delta(t / \varepsilon) e^{G^{\varepsilon}(x, t) / \varepsilon}+r A_{2}, \\
A_{2}=A_{1} \int_{0}^{t / \varepsilon} d t^{\prime} \int_{-\infty}^{\infty} d v \exp \left[v t^{\prime} \partial_{x} G^{\varepsilon}+t^{\prime} \partial_{t} G^{\varepsilon}+O(\varepsilon)\right] \phi\left(t^{\prime}\right) h(v) \Omega_{v}\left(\frac{x}{\varepsilon}, \frac{t}{\varepsilon}, t^{\prime}\right) .
\end{gathered}
$$


As $\varepsilon \rightarrow 0$ the system evolves toward the asymptotic regime where the front is completely developed from its initial condition and travels at constant speed. It is expected then that $P_{v}(x, t)$ converges to the indicator function of the set whose boundary is regarded as the front position that connects the unstable state $P_{v}(x, t)=0$ to the stable state $P_{v}(x, t)=1$. Any initial condition will ensure a front propagating with minimal speed if it has compact support, i.e., has an exponentially bounded tail [26]. We suppose that $J_{v}(x, 0)$ and $P_{v}(x, 0)$ are $A_{1}$ or $A_{2}$ if $x>0$ and zero otherwise, respectively. The condition $G^{\varepsilon}(x, t) \geq 0$ determines the front position when $\varepsilon \rightarrow 0$. Since $e^{-G^{\varepsilon}(x, t) / \varepsilon} \rightarrow 0$ as $\varepsilon \rightarrow 0$ for $G^{\varepsilon}(x, t)>0$, we conclude that the condition $G(x, t)=0$ provides the front position, where $G(x, t)=\lim _{\varepsilon \rightarrow 0} G^{\varepsilon}(x, t)$. The effect of the nonlinear kinetics disappears because $\Omega_{v} \rightarrow 1$ as $\varepsilon \rightarrow 0$ [27]. So that, taking the limit $\varepsilon \rightarrow 0$ in Eq. (14) we find

$$
\left(\begin{array}{cc}
1-\int_{0}^{\infty} \varphi(t) e^{-H t} d t \int_{-\infty}^{\infty} h(v) e^{v p t} & -r \\
-\int_{0}^{\infty} \phi(t) e^{-H t} d t \int_{-\infty}^{\infty} h(v) e^{v p t} d v & 1
\end{array}\right)\left(\begin{array}{l}
A_{1} \\
A_{2}
\end{array}\right)=0,
$$

where $p=\partial_{x} G$ and $H=-\partial_{t} G$ are the momentum and Hamiltonian, respectively. The system of algebraic equations for $A_{1}$ and $A_{2}$ has a nontrivial solution if the determinant of the matrix in Eq. (15) is zero, i.e.,

$$
1=\int_{-\infty}^{\infty} h(v) \hat{\varphi}(H-v p) d v+r \int_{-\infty}^{\infty} h(v) \hat{\phi}(H-v p) d v,
$$

which provides the Hamilton-Jacobi equation. In Eq. (16) we have introduced the notation $\hat{\varphi}(s)=\int_{0}^{\infty} e^{-s t} \varphi(t) d t$. Finally, the front speed $v_{f}$ is obtained by solving the set of equations [1],

$$
v_{f}=\frac{H}{p} \quad \text { and } \quad \frac{d H}{d p}=\frac{H}{p},
$$

together with Eq. (16).

For the CTRW model, the Hamilton-Jacobi equation led to $[1,12]$

$$
1=\hat{\varphi}(H) \Phi(p)+r \hat{\phi}(H),
$$

and so it means that the front speed will be computed from the solution of Eqs. (17) and (18). Next, we will deal with some specific cases in order to compare these theoretical predictions with numerical simulations.

\section{Examples}

(i) Consider first that particles perform flights of duration $\tau$ but their velocities are distributed according to a Gaussian PDF; hence, $h(v)=\pi^{-1 / 2} v_{0}^{-1} e^{-v^{2} / v_{0}^{2}}$ and $\varphi(t)=\delta(t-\tau)$. As the moment-generating function of a Gaussian exists, the front will travel with constant speed. In this case the HamiltonJacobi equation [Eq. (16)] turns into

$$
1=e^{-H \tau+p^{2} v_{0}^{2} \tau^{2} / 4}+r \int_{0}^{\tau} e^{-H t+p^{2} v_{0}^{2} t^{2} / 4} d t,
$$

and the front speed can be computed by solving numerically the set of equations

$$
1=e^{-y v_{f} / v_{0}} e^{y^{2} / 4}+a \int_{0}^{1} e^{-y v_{f} \xi / v_{0}} e^{\xi^{2} y^{2} / 4} d \xi, \quad \frac{d v_{f}}{d y}=0
$$

for $v_{f} / v_{0}\left(y=p v_{0} \tau, a=r \tau\right)$.

On the other hand, it is interesting to compare this result with the equivalent CTRW model. According to Eq. (10), the CTRW counterpart of the example considered here will correspond to a PDF of jump distances $\Phi(x)$ $=\pi^{-1 / 2} v_{0}^{-1} \tau^{-1} e^{-x^{2} / \tau^{2} v_{0}^{2}}$. Since the waiting time distribution is $\varphi(t)=\delta(t-\tau)$ the Hamilton-Jacobi equation [Eq. (18)] reads

$$
e^{H \tau}=e^{p^{2} v_{0}^{2} \tau^{2} / 4}+r \frac{e^{H \tau}-1}{H} .
$$

From Eqs. (17) and (21) we get

$$
\frac{v_{f}}{v_{0}}=\frac{1}{2} \min _{z>0} \frac{z}{\sqrt{\ln \left[e^{z}\left(1-\frac{a}{z}\right)+\frac{a}{z}\right]}},
$$

where $z=H \tau$.

(ii) Let us consider now that particles perform again flights of duration $\tau$ but their velocities are now distributed according to the exponential PDF $h(v)=\left(2 v_{0}\right)^{-1} e^{-|v| / v_{0}}$. The dispersal kernel reads $\Phi(x)=\left(2 \pi v_{0}\right)^{-1} \exp \left(-|x| / \tau v_{0}\right)$, its moment-generating function exists, the Hamilton-Jacobi equation is, from Eq. (16),

$$
1=\frac{e^{-H \tau}}{1-\left(v_{0} p \tau\right)^{2}}+r \int_{0}^{\tau} \frac{e^{-H t}}{1-\left(v_{0} p t\right)^{2}} d t,
$$

and the speed is given by solving numerically the set of equations,

$$
1=\frac{e^{-y v_{f} / v_{0}}}{1-y^{2}}+a \int_{0}^{1} \frac{e^{-y v_{f} \xi / v_{0}}}{1-y^{2} \xi^{2}} d \xi, \quad \frac{d v_{f}}{d y}=0 .
$$

For the CTRW model the Hamilton-Jacobi equation reads

$$
e^{H \tau}=\frac{1}{1-\left(v_{0} p \tau\right)^{2}}+r \frac{e^{H \tau}-1}{H},
$$

and the front speed is then given by

$$
\frac{v_{f}}{v_{0}}=\min \frac{z}{\sqrt{1-\left[e^{z}\left(1-\frac{a}{z}\right)+\frac{a}{z}\right]^{-1}}} .
$$

The comparison of the theoretical expressions for the speed above with numerical results is provided in Fig. 2. In order to check the validity of all the results obtained throughout the paper, we performed a double numerical analysis: (i) we solved numerically the original integral equations [i.e., Eqs. (8) and (9) for the velocity model] and (ii) we performed Monte Carlo simulations that reproduce the processes de- 


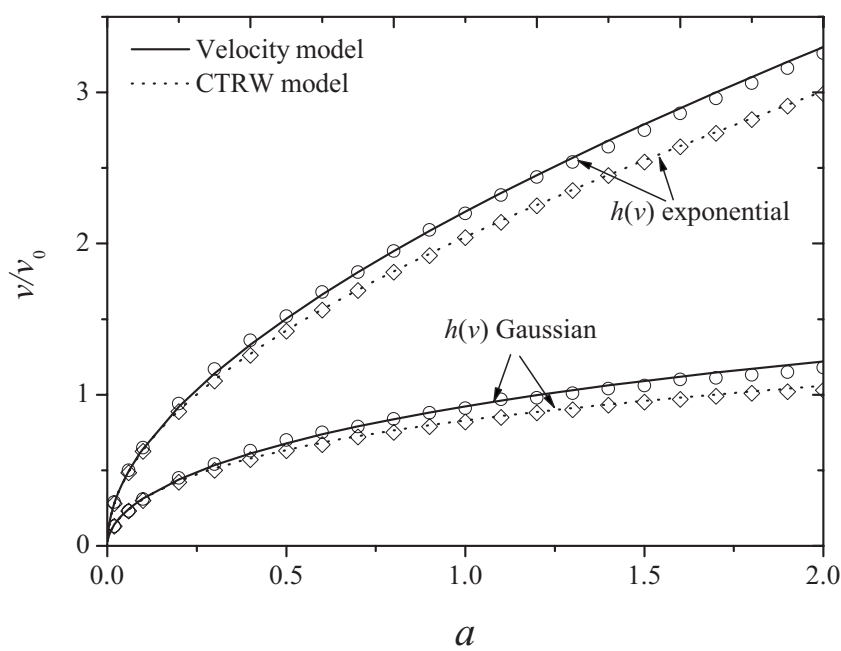

FIG. 2. Comparison for the front speed as a function of $a$ obtained from the velocity model and the CTRW (see Legend) for $\varphi(t)=\delta(t-\tau)$ with Gaussian and exponential speed distribution $h(v)$. The jump kernel corresponding to the CTRW approach is that given by Eq. (10). For simplicity, arbitrary values $\tau=1, v_{0}=1$ are chosen. The corresponding numerical results obtained from Monte Carlo simulations are denoted by circles (velocity model) and diamonds (CTRW).

picted in Fig. 1. In all the cases we observed that the agreement between numerical integration and Monte Carlo simulations was excellent; basically the only differences we found were in the length and the shape of the transitory regime before the wave fronts are formed. So, in the present paper we just show for simplicity the results obtained from Monte Carlo simulations.

The plot in Fig. 2 shows that the agreement between analytical predictions and simulations is excellent for both cases studied here. By comparing the results from the velocity model with those from the CTRW, it must be noted that the speed of fronts obtained from the latter is always smaller, as expected. Also, the differences found between the two models confirm that, even if condition (10) is fulfilled, the equivalence between the jump and velocity approaches breaks down as a result of the interplay between the reaction mechanism and the differences in the density profiles (according to our discussion in Sec. II). As can be seen from the plots, the differences between the models become more important as the reaction parameter $a$ grows so the effect of the reaction process on the system dynamics increases.

\section{ACCELERATED FRONTS}

In this section we want to compute the dependence of the front speed with time when there is acceleration. As explained above this occurs when the dispersal kernel $\Phi(x)$ in Eq. (10) decays asymptotically slower than an exponential function, i.e., its moment-generating function diverges. We center our attention to the situation where both $h(v)$ and $\varphi(t)$ have finite moments and, as a consequence, $\Phi(x)$ also has finite moments [though $\Phi(p)$ diverges]. Consider for example the case where

$$
h(v)=\frac{1}{2 v_{0}} e^{-|v| / v_{0}} \quad \text { and } \quad \varphi(t)=\tau^{-1} e^{-t / \tau} .
$$

From Eq. (10) the dispersal kernel is

$$
\Phi(x)=\frac{1}{v_{0} \tau} K_{0}\left(2 \sqrt{\frac{|x|}{v_{0} \tau}}\right),
$$

where $K_{0}$ is the modified Bessel function of order 0 . Making use of the asymptotic properties of the Bessel function for large $x$, the kernel can be expressed as $\Phi(x)$ $\sim|x|^{-1 / 4} \exp \left(-2 \sqrt{|x| / v_{0} \tau}\right)$ that has finite moments but diverging moment-generating function.

To obtain the speed of the front we linearize Eqs. (8) and (9) around the unstable state because the front speed is determined by the behavior of the front at the leading edge due to the fact that the reaction is monostable $[1,26]$. In consequence, the effects of the nonlinear part of the growth term vanish, as happened in the Hamilton-Jacobi formalism in Sec. IV (we typically use the term pulled front for those cases when this happens). Transforming on space and time to the Fourier-Laplace space $(k, s)$ one gets

$$
\begin{gathered}
J_{v}(k, s)=J_{v}(k, s) \int_{-\infty}^{\infty} \hat{\varphi}(s+i k v) h(v) d v+1+r P_{v}(k, s), \\
P_{v}(k, s)=J_{v}(k, s) \int_{-\infty}^{\infty} \hat{\phi}(s+i k v) h(v) d v
\end{gathered}
$$

By combining the two parts in Eq. (28) one finds

$$
P_{v}(k, s)=\frac{\int_{-\infty}^{\infty} \hat{\phi}(s+i k v) h(v) d v}{1-\int_{-\infty}^{\infty} \hat{\varphi}(s+i k v) h(v) d v-r \int_{-\infty}^{\infty} \hat{\phi}(s+i k v) h(v) d v} .
$$

As we are interested in the asymptotic behavior of the system we consider $s \rightarrow 0$ in Eq. (29). Then, we can expand terms as follows:

$$
\int_{-\infty}^{\infty} \hat{\varphi}(s+i k v) h(v) d v \simeq \Phi(k)+M(k) s+\cdots,
$$

with

$$
M(k)=-\int_{-\infty}^{\infty} h(v) \widehat{t \varphi}(i k v) d v
$$

where $(i k v)$ means Laplace transform where the argument is replaced by $i k v$. Analogously,

$$
\int_{-\infty}^{\infty} \hat{\phi}(s+i k v) h(v) d v \simeq N_{1}(k)+N_{2}(k) s+\cdots,
$$

with 


$$
\begin{gathered}
N_{1}(k)=\int_{-\infty}^{\infty} h(v) \hat{\phi}(i k v) d v \text { and } \\
N_{2}(k)=-\int_{-\infty}^{\infty} h(v) \widehat{t \phi}(i k v) d v .
\end{gathered}
$$

Introducing these expansions into Eq. (29) and inverting by Laplace we obtain, as $t \rightarrow \infty$,

$$
P_{v}(k, t) \simeq A(k) \exp [B(k) t]
$$

with

$$
\begin{gathered}
A(k)=\frac{-M(k) N_{1}(k)-N_{2}(k)[\Phi(k)-1]}{\left[M(k)+r N_{2}(k)\right]^{2}}, \\
B(k)=-\frac{\Phi(k)-1+r N_{1}(k)}{M(k)+r N_{2}(k)} .
\end{gathered}
$$

As $\Phi(x)$ has finite moments we expand Eq. (30) in Maclaurin's series for $k$, following [25]

$$
P_{v}(k, t) \simeq A(k) \Phi(k) \frac{\exp [B(k) t]}{\Phi(k)}=\Phi(k)\left\{A(0) \frac{\exp [B(0) t]}{\Phi(0)}+\sum_{n=1}^{\infty} \frac{k^{n}}{n !} \frac{d^{n}}{d k^{n}}\left[A(k) \frac{\exp [B(k) t]}{\Phi(k)}\right]_{k=0}\right\} .
$$

Inverting by Fourier, it turns into

$$
P_{v}(x, t) \sim \Phi(x) \exp [B(0) t]\left\{1+\frac{\exp [-B(0) t]}{A(0)} \sum_{n=1}^{\infty} \frac{1}{n !} \frac{d^{n}}{d k^{n}}\left[A(k) \frac{\exp [B(k) t]}{\Phi(k)}\right]_{k=0} \frac{1}{\Phi(x)} \frac{d^{n}}{d x^{n}} \Phi(x)\right\} .
$$

If for all $n=1,2, \ldots$ the condition

$$
\lim _{|x| \rightarrow \infty} \frac{1}{\Phi(x)} \frac{d^{n}}{d x^{n}} \Phi(x)=0
$$

holds; then,

$$
P_{v}(x, t) \sim \Phi(x) \exp [B(0) t] \quad \text { as }|x| \rightarrow \infty .
$$

To obtain the front speed we fix $P_{v}(x, t)$ to a constant value of the front tail. The resulting equation $P_{v}(x, t)=$ const relates the front position with time. Differentiating we find

$$
0=\left(\frac{\partial P_{v}}{\partial x}\right) \frac{d x}{d t}+\left(\frac{\partial P_{v}}{\partial t}\right),
$$

and the front speed is given by

$$
v_{f}=\frac{d x}{d t}=-\frac{\partial_{t} P_{v}}{\partial_{x} P_{v}} .
$$

As an example, let us consider three cases. First of all, we take Eq. (26). Clearly, Eq. (27) satisfies condition (32) and for $x \rightarrow \infty$ the dispersal kernel is $\Phi(x) \sim x^{-1 / 4} e^{-\alpha \sqrt{x}}$, where $\alpha$ is a certain constant and $x>0$. From Eq. (33) one has

$$
P_{v}(x, t) \sim x^{-1 / 4} e^{-\alpha \sqrt{x}} e^{r t /(1+r \tau)},
$$

which can be used to get, from Eq. (34),

$$
v_{f}=-\frac{\partial_{t} P_{v}}{\partial_{x} P_{v}}=\frac{4 r x}{(1+r \tau)(1+2 \alpha \sqrt{x})} \sim \sqrt{x} \quad \text { as } x \rightarrow \infty,
$$

so that, integrating $d x / \sqrt{x} \sim d t$, we have finally $x \sim t^{2}$ and

$$
v_{f} \sim t
$$

Second, if we consider

$$
h(v)=\frac{1}{v_{0} \sqrt{\pi}} e^{-v^{2} / v_{0}^{2}} \text { and } \varphi(t)=\tau^{-1} e^{-t / \tau},
$$

the PDF of jumps is

$$
\Phi(x)=\frac{1}{v_{0} \tau \sqrt{\pi}} \int_{0}^{\infty} t^{-1} e^{-t / \tau} e^{-x^{2} / v_{0}^{2} t^{2}} d t
$$

but cannot be calculated explicitly. However, we can apply the Laplace method for integrals with movable maxima [28] to evaluate the asymptotic behavior of Eq. (37). This method is summarized by the following result

$$
\begin{aligned}
\int_{0}^{\infty} f(\lambda, \eta) e^{\lambda S(\eta)} d \eta \simeq & {\left[-\frac{2 \pi}{\lambda S^{\prime \prime}\left(\eta_{0}\right)}\right]^{1 / 2} f\left(\lambda, \eta_{0}\right) e^{\lambda S\left(\eta_{0}\right)} } \\
& +O(1 / \lambda) \text { as } \lambda \rightarrow \infty
\end{aligned}
$$

if $f(\lambda, \eta) e^{\lambda S(\eta)}$ tends to 0 for $\eta \rightarrow 0$ and $\eta \rightarrow \infty, \lambda>0$, and the function $S(\eta)$ has a unique maximum at $\eta=\eta_{0}$. The maximum is movable when its position depends on $x$. The maximum of $-t / \tau-x^{2} / v_{0}^{2} t^{2}$ is attained at $t=\left(x / v_{0}\right)^{2 / 3}(2 \tau)^{1 / 3}$. So that, introducing the change of variables $t=\eta x^{2 / 3}$ into Eq. (37) we find

$$
\Phi(x)=\frac{1}{v_{0} \tau \sqrt{\pi}} \int_{0}^{\infty} \eta^{-1} \exp \left[-x^{2 / 3}\left(\frac{\eta}{\tau}+\frac{1}{v_{0}^{2} \eta^{2}}\right)\right] d \eta .
$$

Now, $S(\eta)=\eta / \tau+1 / v_{0}^{2} \eta^{2}$ and $\lambda=-x^{2 / 3}$. Finally, from Eq. (38) kernel (39) turns into $(x>0)$ 


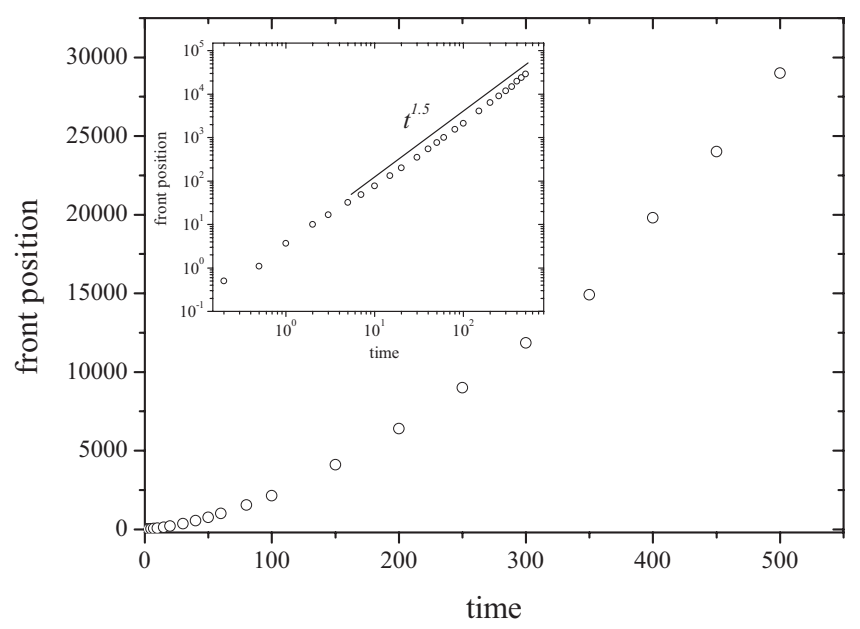

FIG. 3. Time dependence of the front position (in arbitrary units) for the case of $h(v)$ Gaussian and $\varphi(t)=\tau^{-1} e^{-t / \tau}$. Arbitrary values $\tau=0.3, v_{0}=1$, and $a=3$ have been chosen. The log-log plot is given in the inset to observe the corresponding scaling, where the dotted line represents the theoretical scaling in Eq. (35).

$$
\Phi(x) \simeq \frac{1}{\left(v_{0}^{2} \tau^{2} x\right)^{1 / 3}} e^{-3\left(x / 2 v_{0} \tau\right)^{2 / 3}} \quad \text { as } x \rightarrow \infty,
$$

which has also finite moments and satisfies Eq. (32). From Eqs. (33) and (40) we obtain

$$
P_{v}(x, t) \sim x^{-1 / 3} e^{-\alpha x^{2 / 3}} e^{r t /(1+r \tau)},
$$

where $\alpha$ is a certain constant and $x>0$. From Eqs. (34) and (41) one finally obtains

$$
v_{f}=-\frac{\partial_{t} P_{v}}{\partial_{x} P_{v}}=\frac{3 r x}{(1+r \tau)\left(1+2 \alpha x^{2 / 3}\right)} \sim x^{1 / 3},
$$

so that

$$
x \sim t^{3 / 2} \rightarrow v_{f} \sim t^{1 / 2} .
$$

In general, if we consider the PDFs $h(v) \sim e^{-|v|^{n}}$ and $\varphi(t)$ $\sim e^{-t^{m}}$ with $n$ and $m$ higher than 1 , the dispersal kernel is given by $\Phi(x) \sim x^{-n / 2(n+m)} e^{-\alpha x^{n /(n+m)}}(x>0)$, where $\alpha$ is a certain constant. Repeating the same procedure as above we have

$$
x \sim t^{1+m / n} \rightarrow v_{f} \sim t^{m / n} .
$$

In Figs. 3 and 4 we show the behavior of wave fronts obtained from our Monte Carlo simulations for velocity distributions $h(v)$ Gaussian and exponential, respectively. The accelerated nature of the fronts is evident from the plots. The scaling of the front position with time obtained theoretically in Eqs. (35) and (42) is confirmed using a log-log scale (see insets). There one observes that, after a transient regime necessary for the formation of fronts, a power-law scaling for the front position is reached.

To finish, let us consider the third example where the jump velocities are exponentially distributed and $\varphi(t)$ $\sim \sigma^{-1} \exp \left[-(t-\tau)^{2} / \sigma^{2}\right]$. In the limit where $\sigma \rightarrow 0$, the flight duration distribution becomes $\varphi(t)=\delta(t-\tau)$ and we recover the second example studied in Sec. IV where the front propa-

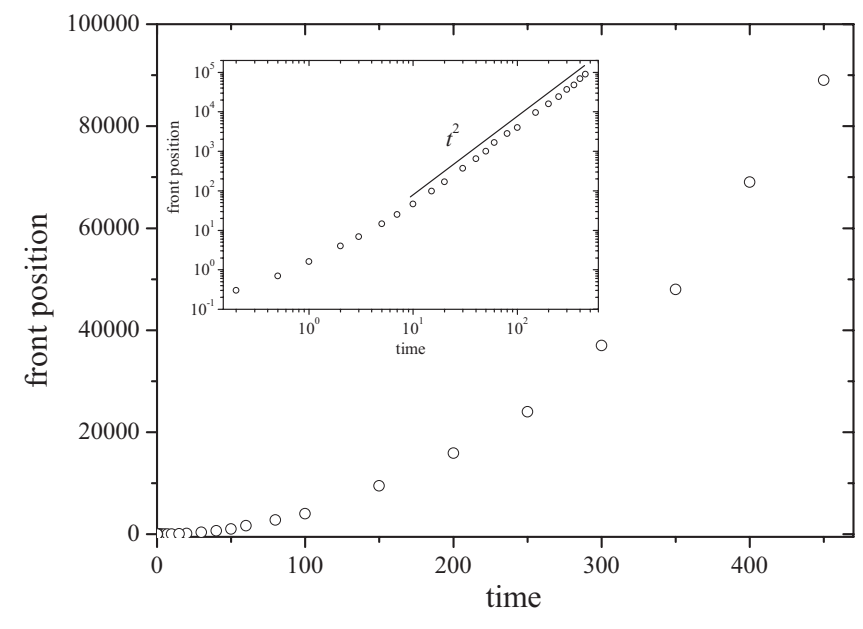

FIG. 4. Time dependence of the front position (in arbitrary units) for the case of $h(v)$ exponential and $\varphi(t)=\tau^{-1} e^{-t / \tau}$. Arbitrary values $\tau=0.3, v_{0}=1$, and $a=3$ have been chosen. The log-log plot is given in the inset to observe the corresponding scaling, where the dotted line represents the theoretical scaling in Eq. (42).

gates with constant speed. Now we show how the method employed in this section also predicts the constant speed of the front in this limit $\sigma \rightarrow 0$. From Eqs. (33) and (34), we obtain

$$
v_{f}=-\frac{\partial_{t} P_{v}}{\partial_{x} P_{v}} \sim-\frac{\Phi(x)}{\Phi^{\prime}(x)} .
$$

Since the dispersal kernel is now

$$
\Phi(x) \sim \int_{0}^{\infty} \frac{1}{\sigma t} e^{-(t-\tau)^{2} / \sigma^{2}} e^{-|x| / \tau v_{0}} d t
$$

and $\lim _{\sigma \rightarrow 0} \Phi(x) \sim e^{-|x| / \pi v_{0}}$ then from Eq. (43) we have

$$
\lim _{\sigma \rightarrow 0} v_{f} \sim-\frac{\lim _{\sigma \rightarrow 0} \Phi(x)}{\lim _{\sigma \rightarrow 0} \Phi^{\prime}(x)}=\text { const }
$$

as expected.

\section{CONCLUSIONS}

We have presented here a kind of reaction-dispersal mechanisms based on the idea that individual particles travel continuously with a speed taken from an arbitrary distribution $h(v)$ instead of making jumps, as is implicitly assumed in most approaches. This mechanism represents the velocity counterpart of the CTRW with reaction, which has already been explored by different authors in the past years [8-11].

Two main results ought to be emphasized from all our discussion and derivations above. First, we have shown that the macroscopic dynamics in a reaction-dispersal mechanism based on velocities and the one based on jumps (CTRW) are different, despite an equivalence exists in the case without reaction [21]. This is a consequence of the differences in the density profiles in each transport model, which become accentuated through the reaction process. Also, a microscopic 
justification for these differences has been sketched in Sec. II. We want to stress that the interest of this result is not merely academic; if there was an equivalence between velocity-based and jump-based approaches, then one could use a tool [Eq. (10) in this case] to translate from one to the other, so that there is no need to study them separately. Since this equivalence breaks down for reaction dispersal, it becomes necessary to specify the microscopic details of the system in order to obtain accurate results for its global dynamics.

Second, we have provided a general criterion (actually adapted from that in [25]) to predict when fronts with constant speed or accelerated are to be expected. From this criterion, we have observed, for example, that a system of particles changing speed with a constant characteristic rate $\tau^{-1}$ [that is, for $\varphi(t)=\tau^{-1} e^{-t / \tau}$ ] and with a speed distribution of the type $h(v) \sim e^{-|v|^{n}}$, with $n \geq 1$, will exhibit accelerated fronts. This includes the case when $h(v)$ is Gaussian or ex- ponential, which are very usual distributions obtained from animal or cell tracking experiments [29-31]. As a whole, it means that accelerated reaction-dispersal fronts should be observed easily if the appropriate experimental setting is available (probably settings based on chemical reactions or cell culture growth would be the best candidates). On the contrary, transport models based on jumps can only lead to accelerated fronts if long-tailed jump length distributions (like stretched exponentials or power-law functions) are considered, which makes experimental observation more difficult to achieve.

\section{ACKNOWLEDGMENTS}

This research has been partially supported by the Ministerio de Ciencia e Innovación under Grant Nos. CGL $2007-$ 60797 (D.C.), FIS 2009-13370-C02-01 (V.M.), and by Generalitat de Catalunya under Grant No. SGR 2009-00164.
[1] V. Méndez, S. Fedotov, and W. Horsthemke, ReactionTransport Systems: Mesoscopic Foundations, Fronts and Spatial Instabilities (Springer-Verlag, Berlin, 2010).

[2] E. Ben-Jacob, I. Cohen, and H. Levine, Adv. Phys. 49, 395 (2000).

[3] V. Ortega-Cejas, J. Fort, and V. Méndez, Ecology 85, 258 (2004).

[4] D. Campos, J. Fort, and J. E. Llebot, Phys. Rev. E 66, 062901 (2002).

[5] D. Campos, J. Fort, and V. Méndez, Theor Popul. Biol. 69, 88 (2006).

[6] R. M. Anderson, R. M. May, and B. Anderson, Infectious Diseases of Humans: Dynamics and Control (Oxford University Press, Oxford, 1991).

[7] R. A. Gatenby and E. T. Gawlinski, Cancer Res. 56, 5745 (1996).

[8] D. Froemberg, H. Schmidt-Martens, I. M. Sokolov, and F. Sagués, Phys. Rev. E 78, 011128 (2008).

[9] D. Campos and V. Méndez, Phys. Rev. E 80, 021133 (2009).

[10] S. Fedotov, Phys. Rev. E 81, 011117 (2010).

[11] E. Abad, S. B. Yuste, and K. Lindenberg, Phys. Rev. E 81, 031115 (2010).

[12] D. Campos, V. Méndez, and V. Ortega-Cejas, Bull. Math. Biol. 70, 1937 (2008).

[13] V. Méndez and J. Camacho, Phys. Rev. E 55, 6476 (1997).

[14] V. Méndez and J. Fort, Phys. Rev. E 60, 6168 (1999).

[15] E. E. Holmes, Am. Nat. 142, 779 (1993).

[16] G. Zumofen and J. Klafter, Phys. Rev. E 47, 851 (1993).

[17] M. Schienbein and H. Gruler, Bull. Math. Biol. 55, 585
(1993).

[18] F. Peruani and L. G. Morelli, Phys. Rev. Lett. 99, 010602 (2007).

[19] D. Campos and V. Méndez, J. Chem. Phys. 130, 134711 (2009).

[20] D. Campos, V. Méndez, and I. Llopis, J. Theor. Biol. 267, 526 (2010).

[21] V. Zaburdaev, M. Schmiedeberg, and H. Stark, Phys. Rev. E 78, 011119 (2008)

[22] M. Schmiedeberg, V. Y. Zaburdaev, and H. Stark, J. Stat. Mech.: Theory Exp. (2009), P12020.

[23] R. Mancinelli, D. Vergni, and A. Vulpiani, Physica D 185, 175 (2003).

[24] D. del-Castillo-Negrete, B. A. Carreras, and V. E. Lynch, Phys. Rev. Lett. 91, 018302 (2003).

[25] J. Medlock and M. Kot, Math. Biosci. 184, 201 (2003).

[26] W. van Saarloos, Phys. Rep. 386, 29 (2003)

[27] In the limit $\varepsilon \rightarrow 0$, Eq. (13) reads $\Omega_{v}\left(\frac{x}{\varepsilon}, \frac{t}{\varepsilon}, t^{\prime}\right)$ $\approx \exp \left[-r t^{\prime} P_{j}^{\varepsilon}(x, t)^{2}\right]=\exp \left[-r t^{\prime} A_{2}^{2} e^{-2 G^{\varepsilon}(x, t) / \varepsilon}\right]$. Since $e^{-G^{\varepsilon}(x, t) / \varepsilon}$ $\rightarrow 0$ as $\varepsilon \rightarrow 0$, finally $\Omega_{v} \rightarrow 1$.

[28] C. M. Bender and S. A. Orszag, Advanced Mathematical Methods for Scientists and Engineers (McGraw-Hill, New York, 1978).

[29] A. Czirók, K. Schlett, E. Madarász, and T. Vicsek, Phys. Rev. Lett. 81, 3038 (1998).

[30] D. Selmeczi, S. Mosler, P. H. Hagedorn, N. B. Larsen, and H Flyvbjerg, Biophys. J. 89, 912 (2005).

[31] D. Valente, I. Golani, and P. P. Mitra, PLoS ONE 2, e1083 (2007). 